\title{
Assessing the Influence of Attitude Towards Mathematics on Achievement of Grade 10 and 12 Female Students in Comparison with Their Male Counterparts: Wolkite, Ethiopia
}

\author{
Engida Melese Simegn ${ }^{1}$, Zeytu Gashaw Asfaw ${ }^{2}$ \\ ${ }^{1}$ Yaberus Wolkite General Secondary and Preparatory School, Wolkite, Ethiopia \\ ${ }^{2}$ School of Mathematical and Statistical Sciences, College of Natural and Computational Sciences, Hawassa University, Hawassa, Ethiopia
}

Email address:

engidumelese@yahoo.com (E. M. Simegn),zeytugashaw@yahoo.com (Z. G. Asfaw)

\section{To cite this article:}

Engida Melese Simegn, Zeytu Gashaw Asfaw. Assessing the Influence of Attitude Towards Mathematics on Achievement of Grade 10 and 12 Female Students in Comparison with Their Male Counterparts: Wolkite, Ethiopia. International Journal of Secondary Education. Vol. 5, No. 5, 2017, pp. 56-69. doi: 10.11648/j.ijsedu.20170505.11

Received: November 21, 2017; Accepted: December 6, 2017; Published: January 10, 2018

\begin{abstract}
Exploring why more males achieve more than females in mathematics at secondary school level when there appear to be no gender differences in the achievement at earlier years is worthy of investigation. The aim of this study was to investigate the influence of attitude towards mathematics on the achievement of female students in comparison with their male counterparts and also to examine the relationship between attitudes and mathematics achievement. The participants of the study were 240 grade ten and 127 grade twelve students of General Secondary and Preparatory School in Wolkite Town. These students were selected using stratified random sampling technique. Attitude towards Mathematics Inventory (ATMI) was used to measure students' attitude, whereas their achievement was measured by the score obtained from Mathematics Achievement Test (MAT). Descriptive Statistics, Multiple Linear Regression Models, Independent - samples $\mathrm{t}$ - test, and Pearson Correlation Coefficient were employed to analyze the collected data by using a Statistical Package for Social Sciences (SPSS) version 20. The results, in both grade levels, unveiled that students had positive attitude towards mathematics but at medium level, however, the level of female students was less than males. The regression models were fit in predicting the contribution of components of attitude towards mathematics scales on students' achievement. In this regard, 42.8\% (Grade 10) and 55.4\% (Grade 12) of the variation in the achievement of students were jointly accounted for by the three variables - the enjoyment, the motivation, and the confidence attitude scales. In both grade levels, no significant gender differences were shown in attitudes towards mathematics but females showed greater decline in attitudes in terms of grade level. However, significant gender differences were specified on achievement. Attitude towards mathematics and students' achievement were significantly correlated $(r=.660 ; p<.001$ (Grade 10) and $r=.735 ; p<.001$ (Grade 12)). Therefore, this study suggests that, the school, the teachers, parents, and concerned bodies have to intervene and design ways in order to enhance students' attitude and performance in mathematics, in particular female students.
\end{abstract}

Keywords: Attitude, Achievement, Component, Scale, Mathematics

\section{Introduction}

Achievement in mathematics and science education at secondary education is critical for every student's lifelong learning or career paths. However, it is an open fact that quite in a large number of General Secondary and Preparatory Schools of Ethiopia, most female students' performance in mathematics is poor. They do not have sufficient knowledge and competence that conform to their grade level, especially in mathematics and science subjects [37]. Likewise, in the school where this study was conducted, achievements of female students in mathematics were poor in comparison with their male counterparts. As an affirmation, in both grade levels, as the data from the school's record office indicated 
female students' yearly academic achievement in mathematics (2012 - 2015) were less than males - for grade ten, 45.99 (Female) and 55.8 (Male); for grade twelve 48.69 (Female) and 59.28 (Male). Moreover, in the same academic years, from the total candidates who sat for grade ten National Examination (Ethiopian General Secondary Education Certificate Examination), out of 1862 female students, $4.13 \%$ got A grade; $26.2 \%$ got B grade; $41.03 \%$ got $\mathrm{C}$ grade; $24.60 \%$ got $\mathrm{D}$ grade; $4.03 \%$ got $\mathrm{F}$ grade and out of 2255 Male students, $9.71 \%$ got A grade; $26.92 \%$ got B grade; $40.31 \%$ got $\mathrm{C}$ grade; $20.67 \%$ got $\mathrm{D}$ grade and $2.39 \%$ got $\mathrm{F}$ grade in mathematics, Regarding grade twelve, from the total candidates who sat for Ethiopian Higher Education Entrance Examination, the average mark for female students was $34.37 \%$ and $39.31 \%$ for their male counterparts in mathematics.

Despite the huge efforts and initiatives that have been taken by the Ethiopian Government to improve the quality of education at all levels of educational institutions, there are some constraints that affect the achievement of students in mathematics at secondary education. Among the challenges, students' negative attitude towards mathematics is the most significant one [13]. Negative attitude towards mathematics can be considered as a serious problem in the teaching learning process of mathematics. As the findings of $[6,22$, 41] show, female students have negative attitude towards mathematics than their male counterparts at secondary education, and also there is a positive relationship between students' attitude and their achievement in mathematics. Moreover, there is a remarkable gender difference in attitude and achievement in mathematics and science subjects in different educational levels [21, 49]. In the school, the researchers from their real observations and eight years experience of teaching mathematics have observed that most female students were not interested in attending mathematics classes. They didn't actively engage and take part in discussions of mathematics lessons. They were not capable of working out activities and exercises in their mathematics text books. They have low participation in most mathematics lessons.

Even though the Ethiopian Government, at Preparatory Level (grade 11 and 12), has placed a great emphasis on the importance of mathematics and science education as an essential component for the sustainable economic growth of the country [18], for example, the government has designed and implemented a policy [36] through which $70 \%$ of the candidates, who passed grade ten National Examinations, enroll in natural science stream to join preparatory program, but the researchers noticed that most students especially females who joined preparatory level of education enroll in social science stream. Besides, most female students, in natural and social science streams, have difficulties in implementing their previous mathematical skills and knowledge to solve mathematical and related problems.

Nevertheless secondary education in Ethiopia has been implemented in two cycles since 2009: grades 9-10 (general secondary) and grades $11-12$ (preparatory), yet, the school runs the teaching learning process combining these two cycles with more than 4000 students. These two stages plays crucial role for students' future career in mathematics and mathematics related subjects. Moreover, grade ten and twelve serve as transition from general secondary to preparatory and from preparatory to university and also can give the overall students' mathematics learning performance and their attitude towards learning mathematics. In addition, it can help mathematics teachers to make use of the findings in their school settings accordingly and as a spring board for other researchers to conduct further study at depth. Furthermore, no research has ever been conducted on female students' attitude towards mathematics and achievement in mathematics and anything that is related to mathematics. Therefore, from this and the aforementioned back ground the authors of this study were initiated also to examine the level of female students' attitude towards mathematics in terms of grade level in comparison with their male counterparts.

Thus, cognizant to the fact that proficiency in mathematics is inevitable at any level of education and currently, as most countries are working hard in order to reach gender equity in mathematics education at secondary level, the researchers carried out the study on the basis of the following specific objectives.

\section{Objectives of the Study}

1. To determine female students' attitude towards mathematics in comparison with their male counterparts.

2 . To investigate whether there is a significant contribution and differences in the attitude component scales on achievement or not.

3. To explore gender differences in attitude and achievement in mathematics.

4. To investigate whether there is a correlation between students' attitude and their achievement in mathematics or not.

5. To examine whether there is a significant difference between students' attitude towards mathematics in terms of grade level with respect to gender or not.

\section{Literature Review}

\subsection{Attitude Towards Mathematics Inventory}

Attitude towards Mathematics Inventory is the most extensively used instruments to measure attitude towards mathematics. It was developed and contributed by [52]. Its final version was made up of 49 items to assess: confidence, anxiety, value, enjoyment, motivation, and parent/teacher expectations. This scale has been the object of extensive studies and modified for application in different situations [22, 33, 41, 55]. Attitudes toward mathematics are very important in the achievement and participation of students in mathematics education. However, under achievement and dislike of mathematics at secondary school levels have increased recognition of the problem of students' attitude, for example, [7, 27, 28]. Thus, in this study, students' attitude 
towards mathematics measured using the following attitude scales.

\subsection{Value of Mathematics}

Mathematics is used as a foundation upon which nearly all natural science and some social science fields of studies are built. It is also more closely related to one's daily life as compared to other subjects. Except one's mother tongue there is no other subject which is more closely related to daily life as mathematics. For that matter, mathematics is a very important required course in secondary school. That is why recommendations of different studies suggested and stressed [27] its importance for students to be proficient in this subject because it plays an important role in career choices and professional development and also under achievement in mathematics limits ones opportunities in competitive professional courses at all tertiary levels. Value/usefulness of mathematics scale has been used as one component of attitude towards mathematics scales [41] to measure students' attitude. This scale, as a component, has a significant contribution on the achievement of students in mathematics [55]. Attitudes of students affected their cognitive activities. By having a positive attitude towards mathematics, the students will feel that mathematics is important so that they will try to improve their mathematics learning achievement. Students who have negative attitudes tend to be difficult to pay attention in math. As [34] pointed out, negative attitudes associated with negative emotional disposition. The disposition of these emotions has an impact on students' behavior to consider the usefulness of mathematics, and do not enjoy math.

\subsection{Mathematics as a Male Domain}

Studies have been done for at least three decades to find out factors that influence female students' achievement in mathematics. Among these factors inhibiting females' pursuit of the study of mathematics was the generally held beliefs that mathematics and science are male domains. In other words, only people with "mathematics minds," mostly men, can do mathematics. Almost all literatures, on gender differences in mathematics, pointed to the commonly held perception that doing mathematics was consistent with a male self - image and inconsistent with female self - image and can affect the learning achievement of mathematics students, especially in high school. As it is reported on [8] on high school students in Ghana, boys get high score on the subscales of ATMI than the girls especially in the total scores of ATMI and on the self-confidence subscales. In this study, mathematics as a male domain scale taken as a predictor variable to check whether this view is persistent in the achievement in mathematics or not.

\subsection{Enjoyment in Mathematics}

Interest in and enjoyment of mathematics is a relatively stable orientation that affects the intensity and continuity of engagement in learning situations, the selection of strategies and the depth of understanding. As the findings of [55] confirmed, this scale has a significant contribution to the achievement of students. In addition, as [57] pointed out that, interest in the subject and performance may be mutually reinforcing and may also be affected by other factors, such as the social back grounds of students and their school. As the findings of [61] indicated in most countries males are statistically significantly more interested in mathematics than females. Even though, interest in mathematics cannot be clearly taken to cause better performance, it is of vale in its own right.

\subsection{Motivation in Mathematics}

Educational Psychologists have long recognized the importance of motivation for supporting students' effective learning. Because initiative is identified as one of the life and career skills necessary to prepare students for post secondary education and the work force [17]. Motivation provides a source of energy that is responsible for why learners decide to make an effort, how long they are willing to sustain an activity, how hard they are going to pursue an activity, and how connected they feel to the activity. Because igniting and sustaining a source of positive energy is so vital to ultimate success.

As [24] highlighted, attitudes are affective responses that accompany a behavior initiated by a motivational state. Attitudes can therefore be linked directly to motivation and provide key information to a better understanding of attitudinal and motivational processes. In the domain of mathematics, studies have pointed out some specific association, as [50] used two sets of items to tap motivation, one related to attendance of school and class the other one participation and preparedness for mathematics classes. On the other hand, other researcher [26], have taken into consideration that motivation affects effort, effort affects result, and positive result lead to an increase in ability. Moreover, on the study with grade ten students also verified that effort is positively and significantly related to mathematics attitudes [26].

\subsection{Confidence in Learning Mathematics}

Studies of learners' self - confidence have been undertaken primarily by psychology and education researchers. These studies have focused particularly on the subject domain of mathematics. Recently, studies have investigated students' self - confidence in their ability on mathematics in numerous countries around the world [39]. Confidence in learning mathematics, or the degree to which a person feels certain of his or her ability to do well in mathematics, has consistently emerged as an important component of gender - related differences. Studies that compared gender differences in mathematics' self confidence have mostly reported that girls had lower confidence in mathematics than boys [39, 48]. Despite such differences, as [39] revealed, the girls' confidence in mathematics improved greatly in classes where girls were 
actively involved in the learning of mathematics.

\subsection{Attitude Towards Mathematics and Achievement}

Attitude towards mathematics plays crucial role in the teaching and learning processes of mathematics. It affects students' achievement. Previous studies on mathematics reported that there is a significant relationship between attitudes and achievement [34, 38, 45]. Attitudes towards mathematics have important role in determining learners' achievement on mathematics, and students with positive attitudes towards mathematics will have high scores in mathematics. In this regard, most recent studies $[7,46,56]$ pointed out that there is a positive correlation between students' attitude towards mathematics and their achievement. Along these lines, [45] revealed students having positive attitude achieved better. [38] in a study with secondary school students showed that those with better academic performance have more positive attitudes than those with poorer academic performance. Contrary to this a study conducted by [23] showed that high achievement can serve to predict a positive attitude towards mathematics, but such an attitude cannot predict higher achievement. This phenomenon is revealed in students at secondary and preparatory level, there are students who have positive attitude towards mathematics but their performance is poor [39]. On the other hand, in terms of gender, as the findings of [28] show that, at secondary school level most of the girls don't actively participate in mathematics classes due to their poor perceptions about mathematics. Furthermore, Girls' positive attitude towards mathematics decline as they grow older. Initially girls have more positive attitude towards mathematics than boys do, but as they continue in school, girls' attitudes become more negative [34].

\subsection{Gender Differences in Attitude and Achievement}

Gender differences are a recurrent theme throughout the literature in academic studies in general and in mathematics studies in particular. Mathematics is often considered to be a domain in which boys are higher achievers, both in terms of attitudes and self - concept. Contrary to this, findings show that, no significant gender difference in the achievement [31, 48]. This similarity in performance between males and females is clear in the Meta - analysis conducted by [31] with data from 242 studies representing 1,286,350 people indicating no gender differences.

There are, however, noticeable differences in the beliefs held by boys and girls. Research has consistently shown that girls have lower math self - concept than boys [51]. In relation to this, results concerning gender differences in attitudes are less consistent than those in self - concept. Some studies have reported significant differences when comparing girls' and boys' attitudes towards mathematics [40, 47]. According to [8], school environment, developmental changes in gender identity, teacher and parent attitudes and believes towards mathematics are factors that may contribute to the differences identified between boys and girls in their attitudes towards mathematics.

Gender differences in mathematics performance has been a great controversy issue in educational domain and research documents show great discrepancies among females and males performance in school mathematics [29]. As long studies history in this area has shows that females had higher performance at lower grade levels $(1-6)$ but as their grade levels increases especially at the end of eighth grade and after gender differences in performance were pervasive - with males having significantly higher achievement than males [40]. On the contrary, as recent researches has shown that the gender differences in mathematics performance are diminishing and these researches indicated that gender differences in mathematics achievement are reducing and shifting, though males were more likely to get higher mean scores [2].

Hypotheses: To achieve the objectives of the study, the following hypotheses were formulated and tested at 5\% level of precision for each grade level -10 and 12:

Hypothesis 1: There is a significant contribution and differences in the attitude towards mathematics component scales on students' achievement.

Hypothesis 2: There is a significant difference between female and male students in terms of their attitude towards mathematics.

Hypothesis 3: There is a significant difference between female and male students in terms of their achievement.

Hypothesis 4: There is a significant correlation between achievement of students and their attitude toward it.

\section{Methodology}

In this study, descriptive survey research design was employed, because it enables the researcher to describe what sort of relationship that would exist among different variables related to the topic under the study and it is also convenient to gather data from a relatively large scale of respondents at a particular time to arrive at better generalization of the existing situation. On the other hand, it involves measurement, classification, analysis, and interpretation of quantitative data, since it is suitable and appropriate to use instruments such as questionnaires, achievement test.

\subsection{Study Area Description}

This study was conducted in Wolkite, the Capital Town of Gurage Zone in South Nations and Nationalities Region States (SNNPRS). Wolkite is about $158 \mathrm{~km}$ from the Capital Addis Ababa. It is almost halfway between Addis Ababa and Jimma. Geographically, it lies between $8^{0} 17^{\prime}$ latitude and $37^{0} 49^{\prime}$ longitude. The school where this study was conducted is Yaberus Wolkite General Secondary and Preparatory School.

\subsection{Sample and Sample Size Determination}

The study was carried out with 240 students from grade 10 of which 124 were female and 116 male. Moreover, 127 
students from grade 12 of which 58 were female and 69 male. These participants were selected using stratified random sampling technique. Sample from each grade level and class were selected by proportional allocation rule. The total sample was calculated employing sample determination formula [12]: $n=\frac{N}{1+N(e)^{2}}$, Where $n$ is the sample size, $N$ is the population size (10 and 12 grade), e is the level of precision $(5 \%)$. Therefore, the sample size for this study was

$$
n=\frac{1818}{1+1818(0.05)^{2}}=327.86 \cong 328
$$

Sample determination for each grade level was obtained as follows:

$$
\begin{aligned}
& \text { Sample for grade 10: } \frac{n_{10}}{n}=\frac{N_{10}}{N} \Longrightarrow n_{10}=n\left(\frac{N_{10}}{N}\right)=328\left(\frac{1209}{1818}\right) \cong 218 \\
& \text { Sample for grade 12: } \frac{n_{12}}{n}=\frac{N_{12}}{N} \Longrightarrow n_{12}=n\left(\frac{N_{12}}{N}\right)=328\left(\frac{609}{1818}\right) \cong 110
\end{aligned}
$$

Where $N_{10}=1209$ (total number of grade ten students), $N_{12}=609$ (total number of grade twelve students), $N=1818$ (target population size)

But $10 \%$ of the sample size for grade ten and $15 \%$ of the sample size for grade twelve were added. These percent were added for contingency purpose and since multiple linear regression model need large sample size and also to meet different statistical analyses criteria.

\subsection{Data Collection Procedure}

Permission to conduct the study was secured by the school administrators. Upon approval, the objectives of the study were described for the school administrators, mathematics teachers, and for the students. Questionnaires, Attitude towards Mathematics Inventory (ATMI) and Mathematics Achievement Test, were administered during the students' free time in the classroom under the supervision of members of mathematics department. The instruments were held at the same time, first ATMI and then MAT. The responses of these instruments for each student were coded.

\subsection{Variables and Measures}

Students' attitude towards mathematics were measured using attitude towards mathematics scales (ATMS) which consists of five component scales - Value (usefulness of mathematics), Male Domain (subject perceived as a male domain), Enjoyment, Motivation, and Confidence scales adapted from [52]. These scales were the independent variables. Each of these scales has eight items in which half of the items were positively stated and the others were negatively stated statements. These instruments were designed to measure the attitude of students and to investigate whether they had statistically significant contribution to students' achievement or not. These scales were scored based on a 1 - 5Likert - type scale and the respondents were asked to respond to each item using a five point Scale such as "Strongly Agree", "Agree", "Undecided", "Disagree", and "Strongly Disagree". The mean score of each attitude scale having 8 items were calculated as, for example, $\frac{5 \times 8}{8}=5$ (maximum mean score if a student is responded strongly agree to positively stated statement and strongly disagree for negatively stated statements). Similarly, the mean of the ATMI (grand mean) were calculated as: $\frac{5 \times 8 \times 5}{40}=5$. Interpretation for mean score for ATMI: If mean score of ATMS was between 1.00 and 3.49 inclusive then it is interpreted as a student had negative attitude towards mathematics; if it is between 3.50 and 5.00 inclusive interpreted as a student had positive attitude. Similar interpretation was applied to the five attitude component scales average as implemented in $[41,22,16]$ researches. Therefore, student's had negative attitude towards mathematics if her/his ATMAS was less than 3.50 and positive if it was greater or equal to 3.50 . In order to verify the internal consistency of items in the ATMS, reliability test has been analyzed using Cronbach's Alpha by SPSS 20. This test was done with reference to the rule stated in [9], yielded

\begin{tabular}{|c|c|c|c|c|}
\hline & \multicolumn{2}{|l|}{ Grade 10} & \multicolumn{2}{|l|}{ Grade 12} \\
\hline & $\mathbf{R}$ & Cronbach's Alpha & $\mathbf{R}$ & Cronbach's Alpha \\
\hline Value of mathematics & $.579-.713$ & $.650-.651$ & $.781-.871$ & $.825-.830$ \\
\hline Perceived maths as male domain & $.659-.768$ & $.713-.717$ & $.531-.724$ & $.629-.635$ \\
\hline Mathematics enjoyment & $.713-.805$ & $.759-.761$ & $.804-.885$ & $.846-.848$ \\
\hline Mathematics motivation & $.731-.816$ & $.775-.776$ & $.805-.885$ & $.847-.849$ \\
\hline Self-confidence in mathematics & $.632-.749$ & $.694-.696$ & $.771-.865$ & $.820-.822$ \\
\hline Attitude towards mathematics & $.782-.849$ & $.815-.817$ & $.922-.953$ & $.937-.939$ \\
\hline
\end{tabular}
the values shown in Table 1.

Table 1. Reliability of ATMS.

Students' performance in mathematics was measured through Mathematics Achievement Tests (MAT) for each grade level. Achievement of students in MAT was the dependent variable. The tests for both grade levels were prepared by the researchers by selecting and modifying the last four years (2012 up to 2015) Ethiopian Government
National Examinations Mathematics Booklets. Besides, the items of the tests covered the first four units of their text books. The items in the tests were based on cognitive levels, i.e., knowledge, comprehension and applications based on table of specification. These tests which were validated by senior mathematics teachers and tested using pilot study 
before the actual tests were administered. Each MAT has 25 multiple choices (each with four options) and was scored out of 100 marks. Based on the MAT score, students in this study were organized in two different achievement groups - Fail with score less than $50 \%$ and Pass - who's scores greater or equal to $50 \%$.

\subsection{Data Analysis}

To analyze the collected data, a number of statistical techniques were employed. The analyses of the contribution of attitude towards mathematics component scales to achievement and correlation among variables - attitudes toward mathematics and achievement were executed through multiple linear regression models. Stepwise regressions were performed to carry out a choice of independent variables from the set of predictors. All the five predictor variables were taken one by one for statistical significance. Independent samples $\mathrm{t}$ - test was used to explore if gender differences on attitudes and achievement existed. These analyses were performed through Statistical Package for Social Science/SPSS version 20.

\section{Results}

Grade 10: Descriptive Statistics of Students Attitude towards Mathematics results, as indicted in Table 2, show that the mean score of VS (Female) $=3.89$ is less than the mean score of VS (Male) $=3.95$ but both are greater than 3.50. This implies that female and male students had positive attitude towards the usefulness of mathematics. The mean score of MDS (Female) $=3.93$ is slightly greater than the mean score of MDS (Male) $=3.91$ which implies that both male and female students didn't perceive mathematics as a male domain subject. The mean score of ES (female) $=3.34$ is less than the mean score of ES (male) $=3.42$, Implies that male students enjoyed doing mathematics more than their female counter parts. Since the mean score of MS (female) $=$ 3.35 and the mean score of MS (male) $=3.37$ are less than 3.50 , the students were not motivated towards learning mathematics. Similar to MS, the mean score of CS (female) = 3.40 and the mean score of CS (male) $=3.36$ are less than 3.50 which was evident that both male and female students were not confident enough in their performance while tackling or doing mathematics problems. The mean score of the ATMI (female) $=3.59$ less than the mean score of the ATMI $($ male $)=3.61$, but both of them had greater than the required mean average.

Grade 12: Similar to grade 10, the descriptive statistics result indicated that, the mean score of the attitude of female students towards the usefulness of mathematics $(M=3.77)$ is less than males' average $(\mathrm{M}=3.99)$ but they are above the required average value. So, these averages indicated that they had positive attitude towards the usefulness of mathematics. The average value of the male domain scale, where students perceived mathematics as a male domain subject, female students average $(M=3.79)$ which is less than the males average $(M=3.88)$, indicated that female students had less attitude than males', they perceived mathematics as a male dominated subject.

Table 2. Descriptive statistics for components of attitude towards mathematics scales and attitude of students.

\begin{tabular}{|c|c|c|c|c|c|c|c|c|}
\hline \multirow{3}{*}{ Variables } & \multicolumn{4}{|c|}{ Grade 10} & \multicolumn{4}{|c|}{ Grade 12} \\
\hline & \multicolumn{2}{|c|}{ Female } & \multicolumn{2}{|l|}{ Male } & \multicolumn{2}{|c|}{ Female } & \multicolumn{2}{|c|}{ Male } \\
\hline & M & SD & M & SD & M & SD & M & SD \\
\hline VS & 3.89 & .39 & 3.95 & .43 & 3.77 & .47 & 3.99 & .43 \\
\hline MDS & 3.93 & .35 & 3.91 & .45 & 3.79 & .56 & 3.88 & .44 \\
\hline ES & 3.34 & .38 & 3.42 & .43 & 3.15 & .57 & 3.26 & .66 \\
\hline MS & 3.35 & .40 & 3.37 & .55 & 3.15 & .52 & 3.27 & .63 \\
\hline CS & 3.40 & .33 & 3.36 & .44 & 3.30 & .56 & 3.30 & .62 \\
\hline ATMS & 3.59 & .28 & 3.61 & .33 & 3.44 & .38 & 3.54 & .48 \\
\hline
\end{tabular}

VS $=$ Value (usefulness) Scales, MDS $=$ Male Domain Scales, ES $=$ Enjoyment Scales,

MS $=$ Motivation Scales, $C S=$ Confidence Scales, $\mathrm{M}=$ Mean, and $\mathrm{SD}=$ Standard Deviation .

But for the overall students' male domain attitude, the average $(M=3.84)$ fulfilled the requirement. Therefore, the whole students didn't perceive mathematics as a male domain subject. That is, they didn't believe that mathematics is only for male students. The enjoyment attitude towards mathematics scale average of female students $(\mathrm{M}=3.15)$ is less than the mean of the males' average $(M=3.26)$. When the average of the enjoyment scale of the whole students (M $=3.21$ ) is examined it is below the average [3.50] implied that students didn't enjoy doing mathematics. Motivation scale's average of both gender are $\mathrm{M}$ (female) $=3.15$ and $\mathrm{M}$ $($ Male $)=3.28$ as indicated in Table 2 . Therefore, this result shows they didn't have motivation for doing mathematics. In other words, they were not motivated to engage and willing to sustain activities in learning mathematics. The confidence scale' mean score of both gender are almost equal but below 3.50. Meaning both females and males didn't have confidence in their performance while tackling or doing mathematics. In general, the mean score of attitude towards mathematics scale, ATMS, of females' $(M=3.44)$ less than males' $(M=3.54)$, indicated that male students to some extent had positive attitude towards mathematics than females. But their mean score are almost around 3.50. Therefore, this result shows these students had medium attitude towards mathematics.

Hypothesis 1: There is a significant contribution and differences in the attitude towards mathematics component scales on students' achievement. 
In order to determine how much of the variance in the score of students' mathematics achievement test could be explained by the above mentioned attitude component scales, multiple regression analysis was utilized. The results of this analysis, as Table 3 summarizes, in both grade levels, the sig. $\mathrm{p}$ - values for the three predictors: ES, MS, and CS are less than .05 (significant) indicated that there is a sufficient evidence to accept the hypothesis. In addition, since the other two predictor variables value of mathematics and subject perceived as a male domain attitude scales were deleted due to their insignificant contribution by stepwise method, the results of the analysis confirmed the anticipation in the above hypothesis.

Table 3. The contribution of each component attitude scale on achievement.

\begin{tabular}{llllll}
\hline \multicolumn{2}{l}{ Model Summary } & & & & \\
\hline Model & Grade & Multiple R & R Square & Adjusted R Square & St. error of estimate \\
\hline \multirow{2}{*}{3} & 10 & .654 & .428 & .420 & 8.730 \\
& 12 & .744 & .554 & .543 & 7.949 \\
\hline
\end{tabular}

Predictors: (constant), Average of E - scale, Average of M - scale, and Average of C - scale.

\begin{tabular}{|c|c|c|c|c|c|c|c|}
\hline \multicolumn{8}{|c|}{ ANOVA } \\
\hline Model & Grade & & Sum of Squ. & Df & Mean squ. & $\mathbf{F}$ & Sig. \\
\hline \multirow{6}{*}{3} & \multirow{3}{*}{10} & Regression & 13432.777 & 3 & 4477.592 & \multirow[t]{3}{*}{58.749} & \multirow[t]{3}{*}{.000} \\
\hline & & Residual & 17986.956 & 236 & 76.216 & & \\
\hline & & Total & 31419.733 & 239 & & & \\
\hline & \multirow{3}{*}{12} & Regression & 9651.303 & 3 & 3217.101 & \multirow[t]{3}{*}{50.917} & \multirow[t]{3}{*}{.000} \\
\hline & & Residual & 7771.563 & 123 & 63.183 & & \\
\hline & & Total & 17422.866 & 126 & & & \\
\hline
\end{tabular}

Dependent Variable: Mathematics Achievement Test.

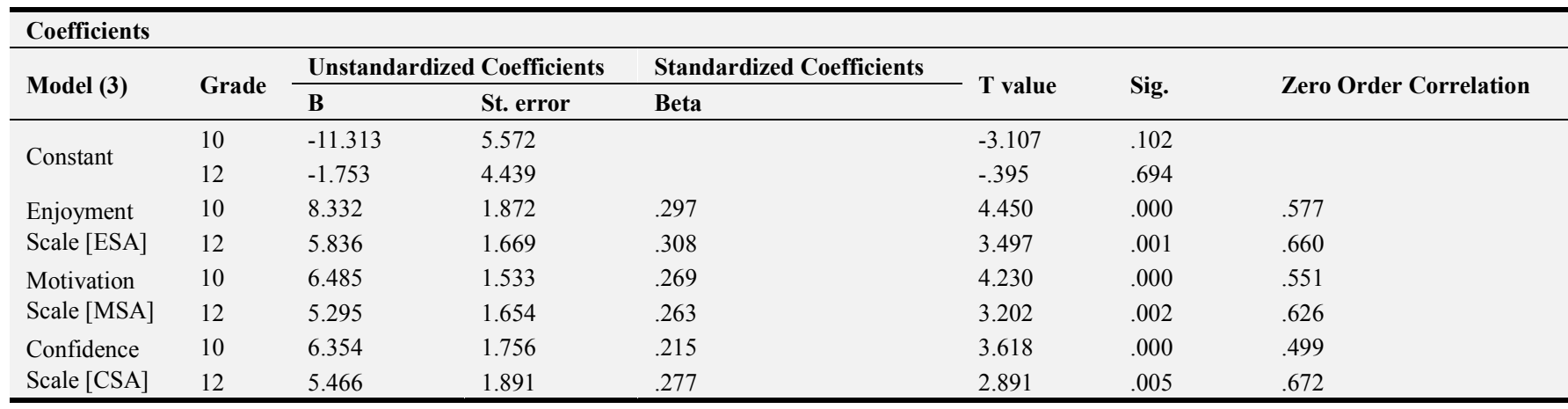

Dependent Variable: Mathematics Achievement Test.

As reflected in Table 3 the regression equations can be expressed as:

Grade $10: \mathrm{Y}=-11.313+8.332 \mathrm{ESA}+6.485 \mathrm{MSA}+$ 6.354CSA,

Grade $12: \mathrm{Y}=-1.753+5.836 \mathrm{ESA}+5.295 \mathrm{MSA}+$ 5.466CSA,

Where $\mathrm{Y}=$ Mathematics Achievement Test Score, ESA = Enjoyment Scales Average, MSA = Motivation Scales Average and CSA $=$ Confidence Scales Average

Grade 10: Table 3 shows that, the three components of attitude towards mathematics scale ES, MS, and CS have significant contribution to the achievement of the students in MAT, this is shown by the multiple $\mathrm{R}$ value of 0.654 which signifies moderate positive correlation $[11, P .8$ of 28$]$. The $R^{2}=0.428$ suggests that $42.8 \%$ of the variation in the MAT score is jointly accounted for by the three attitude scales. $\left(F_{(3,239)}=58.749, \mathrm{p}<0.05\right)$ indicated that the multiple correlation $\mathrm{R}=0.654$ was significant. It implies that the joint variation of the three attitude scales in explaining the variation in the achievement was significant. The $t-$ test results however show that only ES, MS and CS have unique significant contribution on the achievement.

The percent of the contribution of each attitude variables $\mathrm{E}, \mathrm{M}$, and $\mathrm{C}$ was obtained by the equation that links $R^{2}=.428$ as indicated in [10] analysis:

$$
R_{0 V E M C}^{2}=\operatorname{Beta}_{E} r_{0 E}+\text { Beta }_{M} r_{0 M}+\text { Beta }_{C} r_{0 C}
$$

Where, $\beta s$ are the coefficients of independent variables attitude component scales, $r_{s}$ are the zero order correlations. Zero - Order correlation coefficients (often called validity coefficients) [30]. It is the Pearson correlation between each predictor and dependent variable[14,p.6]. If all predictors are uncorrelated, $r_{s}$ values partitioned $R^{2}$ value or, equivalently, they added up to the model $R^{2}$ [30]. Therefore, this $R^{2}$ value can be expressed in terms of the extent of the contribution of each of the three attitude scales to the achievement as:

$$
\begin{gathered}
.428 \times 100 \%=(.297 \times .577+.269 \times .551+.215 \times .499) 100 \% \\
42.8 \% \approx 17.14 \%+14.82 \%+10.73 \%
\end{gathered}
$$


Which means the three attitude scales variables ES, MS, and CS contribute $17.14 \%, 14.82 \%$, and $10.73 \%$ respectively to the achievement of students in mathematics.

Moreover, in the above equation, unstandardized coefficients show that the higher the coefficient the ESA had, the greater the contribution to the achievement scores in MAT. Because it is reflected from B - Values: B (ESA) = 8.332 whereas $\mathrm{B}(\mathrm{CSA})=6.354$. Therefore, the ES had a greatest contribution $(17.14 \%)$ that produced the biggest variance in the achievement of students.

Grade 12: Table 3 summarizes that the three attitude scales ES, MS, and CS have significant effect on the achievement of students in MAT. This is shown by the multiple $\mathrm{R}$ value of .744 which signifies strong positive correlation. The $\mathrm{R}-$ square or the coefficient of determination value .554 indicated that $55.4 \%$ of variation in the achievement score was jointly accounted for by the three attitudes component scales. The $\mathrm{F}$ - ratio, 50.917 was found to be significant at the .05 level of significance. It implies that the joint variation of the items in explaining the variation in the achievement score was significant. For this model, ES, MS, and CS have significant contribution, since their $\mathrm{t}$ - test values $(\mathrm{p}<.05)$. The percent of the contribution of each attitude variables $\mathrm{E}, \mathrm{M}$, and $\mathrm{C}$ was obtained by the equation that links $R^{2}=.554$ as indicated in grade 10 analysis as:

$$
\begin{gathered}
.554 \times 100 \%=(.308 \times .660+.263 \times .626+.277 \times .672) 100 \% \\
55.4 \% \approx 20.33 \%+16.46 \%+18.61 \%
\end{gathered}
$$

Therefore, ES had the greatest contribution $20.33 \%$ and MS has the smallest $16.46 \%$.

Hypotheses 2 and 3: There is a significant difference between female and male students in terms of their attitude and achievement.

In order to investigate gender differences in attitude and achievement, independent samples $\mathrm{t}$ - test analysis was implemented. The results of this analysis on participants' attitude towards mathematics and their average scores of mathematics achievement test in terms of gender are given as follows

Grade 10: Table 4 shows no significant gender differences were specified in the attitudes of students towards the components of attitude scales such as value of mathematics, subject considered as a male domain, enjoyment in mathematics, motivation in mathematics, and self confidence in mathematics scales. Because their $\mathrm{t}-$ values $\mathrm{t}$ $(238)=-1.166$ with Sig. $(p)=.245, \mathrm{t}(238)=.309$ with sig. (p) $=.758, \mathrm{t}(238)=-1.464$ with Sig. $(\mathrm{p})=.144$, and $\mathrm{t}(238)=-$ .304 with Sig $(p)=.762, t(238)=.862$ with Sig $(p)=.390$ respectively are greater than .05 . Therefore, this result led to reject hypothesis 2. As to students' attitude towards mathematics, Table 4 shows, female and male students had similar attitude towards mathematics. This means, statistically female students' positive attitude towards mathematics $(\mathrm{M}=3.5869, \mathrm{SD}=.2809)$ had no significant difference from males $(\mathrm{M}=3.6057, \mathrm{SD}=.3314)$, because the Sig (p) value is greater than .05 . Hence, this result again led to reject hypothesis 2 .

In addition, Table 4 indicates that there was no significant gender difference in MATSA. That is, the mean score of the female students $(\mathrm{M}=51.32, \mathrm{SD}=10.98)$ had a significant difference with male students MATSA $(\mathrm{M}=54.69$, SD = 11.898), because the Sig (p) value is .024 that led the researchers to accept hypothesis 3 .

Grade 12: Female participants got lower means in all variables. However, the difference did not reach significance for attitude and all attitude components except for usefulness/value of mathematics and achievement. Descriptive statistics on the mean differences indicated that statistically significant differences were noticed in the value of mathematics and achievement, female participants getting the lower means $(\mathrm{M}=49.38, \mathrm{SD}=9.553)$ and $(\mathrm{M}=3.7725$, $\mathrm{SD}=.4725)$ than their male counterparts $(\mathrm{M}=54.38, \mathrm{SD}=$ $12.967)$ and $(\mathrm{M}=3.9879, \mathrm{SD}=.4313)$ respectively. This is because of $\mathrm{t}(125)=-2.432, \operatorname{Sig}(\mathrm{p})=.016$ for achievement and $\mathrm{t}(125)=-2.683, \operatorname{Sig}(\mathrm{p})=.008$ for participants perceived the usefulness of mathematics. Therefore, this result led us to accept hypothesis 3 .

Table 4 shows, no significant gender differences were specified in the attitudes of students towards the components of attitude scales such as subject considered as a male domain, enjoyment in mathematics, motivation in mathematics, and self - confidence in mathematics scales. Because their $\mathrm{t}-$ values $\mathrm{t}(125)=-1.083$ with Sig. $(\mathrm{p})=.281, \mathrm{t}$ $(125)=-.990$ with Sig. $(p)=.324, t(125)=-1.159$ with Sig. $(\mathrm{p})=.249$, and $\mathrm{t}(125)=.030$ with $\mathrm{Sig}(\mathrm{p})=.976$ respectively are greater than .05 . Hence, as a result of this hypothesis 2 were rejected. Meaning, statistically they didn't have different attitude towards these attitudes. Moreover, as their attitude as a whole considered in terms of gender, statistically there was no significant difference on the attitude towards mathematics between female and male students. Because its $t$ - value $\mathrm{t}(125)=-1.373$ with Sig. (p) $=.172$ which was greater than .05 . As to the comparison of achievement, Table 4 shows, there was statistically significant difference between the mean score of female students in mathematics achievement test $(\mathrm{M}=49.38, \mathrm{SD}=9.553)$ and the mean score of male students $(\mathrm{M}=54.38, \mathrm{SD}=12.967)$.

Concerning the status of students' attitude towards mathematics in terms of grade level with respect to gender, a decline in attitude were observed in both grade level and in both gender. However, statistically there was a significant difference on the attitude between grade ten and grade twelve female students, $\mathrm{t}(180)=2.993$ with Sig. $(p)=.003$, whereas, no significant difference was specified between grade ten and grade twelve male students, $\mathrm{t}(183)=1.035$ with Sig. (p) $=.302$. This result indicated that as female students' progress in their grade level no attitude enhancement was seen, grade ten female: $\mathrm{M}=3.5869$ with $\mathrm{SD}=.2809$ and grade twelve female: $\mathrm{M}=3.4360$ with $\mathrm{SD}=.3833$, but in case of male students, despite the decline their attitude was better than their female counterparts in both grade levels, grade ten male: $\mathrm{M}=3.6057$ with $\mathrm{SD}=.3314$ and grade twelve male: $\mathrm{M}$ $=3.5437$ with $\mathrm{SD}=.4826$. 
Table 4. Independent Samples t-test of Attitude towards Mathematics and Mathematics Achievement Test Scores.

\begin{tabular}{|c|c|c|c|c|c|c|}
\hline Variables & Grade & G & Mean & SD & $t$-value & Sig. (2 - tailed) \\
\hline \multirow{4}{*}{ Value Scales [VS] } & \multirow{2}{*}{10} & $\mathrm{~F}$ & 3.8931 & .38941 & \multirow[t]{2}{*}{-1.166} & \multirow[t]{2}{*}{.245} \\
\hline & & M & 3.9547 & .42743 & & \\
\hline & \multirow{2}{*}{12} & $\mathrm{~F}$ & 3.7725 & .47255 & \multirow[t]{2}{*}{-2.683} & \multirow[t]{2}{*}{.008} \\
\hline & & M & 3.9879 & .43134 & & \\
\hline \multirow{4}{*}{ Male Domain Scales [MDS] } & \multirow{2}{*}{10} & $\mathrm{~F}$ & 3.9279 & .35421 & \multirow[t]{2}{*}{.309} & \multirow[t]{2}{*}{.758} \\
\hline & & M & 3.9117 & .45440 & & \\
\hline & \multirow{2}{*}{12} & $\mathrm{~F}$ & 3.7870 & .55814 & \multirow[t]{2}{*}{-1.083} & \multirow[t]{2}{*}{.281} \\
\hline & & M & 3.8832 & .44314 & & \\
\hline \multirow{4}{*}{ Enjoyment Scales [ES] } & \multirow{2}{*}{10} & $\mathrm{~F}$ & 3.3401 & .38163 & \multirow[t]{2}{*}{-1.464} & \multirow[t]{2}{*}{.144} \\
\hline & & M & 3.4173 & .43431 & & \\
\hline & \multirow{2}{*}{12} & $\mathrm{~F}$ & 3.1538 & .56569 & \multirow[t]{2}{*}{-.990} & \multirow[t]{2}{*}{.324} \\
\hline & & M & 3.2634 & .66430 & & \\
\hline \multirow{4}{*}{ Motivation Scales [MS] } & \multirow{2}{*}{10} & $\mathrm{~F}$ & 3.3546 & .40124 & \multirow[t]{2}{*}{-.304} & \multirow[t]{2}{*}{.762} \\
\hline & & M & 3.3733 & .54695 & & \\
\hline & \multirow{2}{*}{12} & $\mathrm{~F}$ & 3.1546 & .51827 & \multirow[t]{2}{*}{-1.159} & \multirow[t]{2}{*}{.249} \\
\hline & & M & 3.2750 & .63288 & & \\
\hline \multirow{4}{*}{ Confidence Scales [CS] } & \multirow{2}{*}{10} & $\mathrm{~F}$ & 3.4034 & .33455 & \multirow[t]{2}{*}{.862} & .390 \\
\hline & & M & 3.3601 & .43924 & & \\
\hline & & $\mathrm{F}$ & 3.3032 & .56413 & .030 & .976 \\
\hline & 12 & M & 3.3000 & .62460 & & \\
\hline & 10 & $\mathrm{~F}$ & 3.5869 & .28093 & -.476 & .634 \\
\hline Attitude towards Math Inventory [ATMI] & 10 & M & 3.6057 & .33142 & & \\
\hline 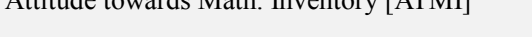 & 12 & $\mathrm{~F}$ & 3.4360 & .38332 & -1.373 & .172 \\
\hline & 12 & $\mathrm{M}$ & 3.5437 & .48263 & & \\
\hline & 10 & $\mathrm{~F}$ & 51.32 & 10.984 & -2.280 & .024 \\
\hline Mathematics Achievement Test Score [MATS] & 10 & M & 54.69 & 11.898 & & \\
\hline & 12 & $\mathrm{~F}$ & 49.38 & 9.553 & -2.432 & .016 \\
\hline & 12 & M & 54.38 & 12.967 & & \\
\hline
\end{tabular}

Grade $10-$ Female $=124$, Male $=116 ;$ Grade $12-$ Female $=58$, Male $=69$.

Hypothesis 4: There is a significant positive correlation between achievement of students and attitude toward it.

In order to explore this hypothesis Pearson correlation coefficient analysis was utilized. Different Authors suggested different interpretations of the values of the correlation coefficients; however, the researcher used [53,p.37] roughly categorized $\mathrm{r}$ values as, $0<\mathrm{r} \leq .35$ (or $-.35 \leq \mathrm{r}<0$ ) are generally considered to represent low or weak correlation, $\mathrm{r}=.36$ to $\mathrm{r}$ $=.67$ or $\mathrm{r}=-.36$ to $\mathrm{r}=-.67$ as moderate correlations, $\mathrm{r}=.68$ to $\mathrm{r}=.89$ or $\mathrm{r}=-.68$ to $\mathrm{r}=-.89$ as strong or high correlations, and as[11, p. 8 of 28] indicated that $r$ values very close to 1 as high positive correlation.

Table 5. The Pearson Correlation Coefficients among the variables.

\begin{tabular}{|c|c|c|c|c|c|c|c|c|}
\hline Grade & Variable & Gender & VSA & MDSA & ESA & MSA & CSA & ATMSA \\
\hline \multirow{2}{*}{10} & \multirow{4}{*}{ MATSA } & Female & $.342 *$ & $.409 *$ & $.664 *$ & $.507 *$ & $.550 *$ & $.661 *$ \\
\hline & & Male & $.263^{*}$ & $.466^{*}$ & $.548^{*}$ & $.588^{*}$ & $.475^{*}$ & $.666^{*}$ \\
\hline \multirow{2}{*}{12} & & Female & $.356^{*}$ & $.370^{*}$ & $.497 *$ & $.435 *$ & $.641^{*}$ & $.649 *$ \\
\hline & & Male & $.598^{*}$ & $.464 *$ & $.746^{*}$ & $.717 *$ & $.720 *$ & $.771 *$ \\
\hline
\end{tabular}

Correlation is significant at $\mathrm{p}^{*}<0.05(1-$ tailed $)$.

Table 5 shows that, in both grade levels, the correlation between achievement (MATSA) and attitude of students towards mathematics (ATMSA) for both female and male students at .05 level of precision are statistically significant. In addition, the strength of the correlation between achievement and the other variables was better in female students than males. When considering grade twelve, almost weak correlation between VSA, MDA and achievement were specified in female participants than males. Moreover, strong positive correlations were found between EVS, MVS, CVS and MATS; moderate positive correlations were observed between VS, MD and MATS on male participants. Since slightly strong positive correlation was specified in male students than females in both grade levels. This implies that at the 0.05 significance level, positive and significant correlation exists between attitude of the students and their achievements. Therefore, the null hypothesis was accepted.

\section{Discussion}

Positive attitude towards mathematics is needed to influence one's willingness to learn and achieve in mathematics regardless of gender. But as the result of this study indicated that the mean score of attitude towards mathematics scale of female students in both grade levels were less than their male counterparts. However, their mean score were around the average level. These values indicated that, to some extent male students had better attitude towards 
mathematics than females. These findings for grade ten were in-line with $[1,22]$, because in the findings of [22] the mean score of female students' attitude was less than their male counterparts in which attitudes had significant effect on students achievement in the government schools but not with [6]. The result of the study for grade twelve is relevant with the prior study [34]. Concerning attitude towards mathematics in terms of grade level, the mean score of each component scales of grade ten female students was greater than grade twelve females. This result is confirmed by [34], because, the result of this study revealed that females showed a continuous decline in attitudes the further they progressed in grade levels. Similarly, the mean score of grade ten male students was greater than grade twelve males. In general, as different findings found out that students attitude towards mathematics influenced by many factors such as by personal interests and teaching methods [57], teachers attitude towards mathematics [35], teachers and the social support of peers [34], poor teaching environment, inadequate self - practice, and students' poor back ground [58] and the likes.

As aforementioned, one of the objectives of this study was to investigate the individual contribution of each attitude predictor on the achievement of students. As the regression analysis for both grade levels confirmed the existence of statistical differences in the contribution of component attitude scales to the achievement which was in-line with the researchers claim. In other words, the result confirmed the hypothesis. The positive value of the coefficients for each attitude scales indicates positive relationship. An increase in the average value of each of these predictors will show an increase in the achievement scores of students. It implied that the more students had positive attitude towards mathematics, the more likely they could achieve. When considering grade levels, as the findings for grade ten indicated $42.8 \%$ of variation in the achievement score was jointly accounted for by enjoyment, motivation, and confidence attitude scales. The remaining $57.2 \%$ of the achievement of students in mathematics was affected by other attitude scales which were not included in this study. In addition, achievement of students might be affected by educational environment factors such as inadequate teaching motivation, poor assessment, poor teaching methods, and teachers' attitude towards mathematics [39, 44]. As the findings for grade twelve indicated $55.4 \%$ of variation in the achievement score was jointly accounted for by enjoyment, motivation, and confidence attitude scales. The remaining $44.6 \%$ of the achievement of students in mathematics might be affected by other factors such as inadequate teaching motivation, poor assessment, poor teaching methods [2], and teachers' attitude towards mathematics or their job [39, 42, 44] When the objective of this study expressed in terms of grade levels, individual contribution of each attitude predictor on the achievement of students, as it can be seen in the above discussions, were greater in grade twelve as compared to grade ten. However, the type of the attitude predictors were similar, namely ES, MS, and CS. As the regression analysis in Table 3 indicated, the significant contribution of ES, MS, and CS to the achievement of students were greater in grade twelve than grade ten, because $54.4 \%$ of variation in the achievement score was accounted for by these attitude scales in grade twelve whereas in grade ten was $42.8 \%$. This implies that the achievement of grade twelve students were more influenced by students' attitude towards mathematics than grade ten students. As the result of this study for grade ten indicated, no significant gender differences were specified in the attitudes of students towards the components of attitude scales such as value of mathematics, subject considered as a male domain, enjoyment in mathematics, motivation in mathematics, and self - confidence in mathematics scales. Regarding the relevance of the results of this study, the insignificant difference between female and male students in the usefulness of mathematics scale is in line with the findings of [38] who were conducted a research on 200 secondary school students showed that, the students had medium and positive attitude towards the usefulness of mathematics and no gender difference in their attitudes. Concerning the result of this study on self - confidence of students in mathematics is contrary with the findings of [46], because the result of this study indicated that self confidence in mathematics lead to differences in perceptions about mathematics. This in turn may lead to differences in attitude towards mathematics and learning mathematics which have bearing on performance, but concurred with the findings of the study by [38], since the result of this study showed that the students had medium and positive attitude on self - confidence in mathematics and no gender difference. Despite the level of students' motivation towards learning mathematics, the result of this study is in - line with the findings by [34], because the result of this study indicated that no gender differences were specified on students' motivation. However, motivation related variables are the main predictors of attitude towards mathematics and highly significant in understanding these attitudes. Referring to the study carried out by [54], the result of this study is in contrary, since the result of [54] showed that gender difference is significant when the impact of motivation on academic achievement is compared in female and male students. As to students' attitude towards mathematics, Table 4 shows, female and male students had similar attitude towards mathematics and no significance difference was specified between the two genders which is concurred with the findings of [19], since the result of their study revealed that statistically no significant difference between female and male students, but against with the outcome of the study by [46] who were conducted a study on 124 randomly selected secondary school students in South Africa that showed the existence of significant differences in the perceptions and beliefs about mathematics between female and male students.

In grade ten, even though, the mean score of both female and male students in MAT were satisfactory, this result is in line with [22] study conducted on Anxiety, Attitude towards mathematics and mathematics achievement of 307 tenth grade Government and Private Schools students in Kolfe Keranio Sub city of Addis Ababa, Ethiopia and with [2] 
findings, since their result indicated that performance is a function of orientation, not gender, But contrary to the findings of [39] conducted a study on attitude towards mathematics and achievement in mathematics among grade 9 and 10 secondary level boys and girls. They used 200 boys and 300 girls' samples taken from four schools. On the bases of their examination results their findings showed that, girls achieved better results as compared to boys.

For grade twelve, no significant gender differences were specified in the attitudes of students towards the components of attitude scales such as subject considered as a male domain, enjoyment in mathematics, motivation in mathematics, and self - confidence in mathematics scales. For instance, in mathematics domain students with high motivation had a more positive attitude towards mathematics, despite their low motivation (see table 4) the result of this study was in-line with the findings of [34] where the students had positive attitude towards mathematics. They conducted their study on utilizing "Intrinsic Motivation Inventory", but in contrast with [54] findings. As their attitude as a whole considered in terms of gender, statistically there was no significant difference on the attitude towards mathematics between female and male students, but their attitude were around the average value. As [34] results indicated that despite their positive attitude towards mathematics, as female students grade level increases their attitude decline. Therefore, the finding of this research is not in-line with [34] as far as the methodology and other related things were taken into account. As the findings of this study concerning achievement of MAT indicated the existence of statistically significant difference between female and male students which is in-line with the findings of [43] who conducted a research on gender difference in mathematics on 857 grade twelve students (355 boys and 502 girls) in which females' achievements were lower than males.

Concerning the status of students' attitude towards mathematics in terms of grade level with respect to gender, a decline in attitude were observed in both grade level and in both gender. However, statistically there was a significant difference on the attitude between grade ten and grade twelve female students, whereas, no significant difference was specified between grade ten and grade twelve male students. This result indicated that as female students' progress in their grade level no attitude enhancement was seen, but in case of male students, despite the decline their attitude was better than their female counterparts in both grade levels. This result, in case of female students, was in - line with the result of [34] but not in case of male students, because their findings revealed that the decline in attitude was seen in female students only.

One of the objectives of this study was to investigate whether there is a significant relationship between attitude and achievement, results of this study for grade ten is not relevant with the prior study done by [39] who conducted a study on attitude towards mathematics and academic achievement in mathematics among secondary level boys and girls of grade nine and ten. The results of this study indicated that attitude towards mathematics and achievement in mathematics did not go together, But the results of this study is supported by the findings of [16]. Statistically positive and significant correlations were existed between attitude components and achievement. Regarding to the strength of the relationship between components of attitude scales and achievement, moderate correlations were seen in tenth grade except the relation between achievement and value of mathematics scales of male participants, because it is a weak correlation $\left(.263^{*}\right)$. Regarding to the strength of the relation between achievement and attitude, there was strong positive correlation between achievement and attitude towards mathematics. As to the strength of the correlation compared with respect to gender, slightly strong positive correlation was specified in male students than females in both grade levels which are supported by the findings of the study conducted by [16].

\section{Conclusions}

Based on the results and the analyses, the following conclusions were drawn.

In both grade levels, the attitudes of students were found to be at medium level. However, subject perceived as valuable and male domain attitudes were above the medium level, whereas, enjoyment, motivation, and confidence attitudes in mathematics were below the medium level. As a whole female students' attitude level at each component of attitude was less than their male counterparts.

In both grade levels, the contributions of the three variables - enjoyment in mathematics, motivation towards learning mathematics, and self - confidence in mathematics to the achievement were statistically significant, but higher for grade twelve. Therefore, it can be inferred that such attitudes are inevitable for the improvement of students' performance in the subject, in particular for female students.

Even though, it appeared in both grade levels, female students had a slightly lower mean attitude towards mathematics inventory than their male counterparts, no gender differences were identified. In terms of their achievement, significant gender differences were observed as anticipated by the authors. Despite the gap, the disparities of the achievement between the two genders indeed similar to the prior students' data obtained from the school's record office.

The positive correlation, in both grade levels, between students' attitude and their achievement in mathematics demonstrated that attitude plays an important role in female students' performance despite the medium score of their attitude and poor achievement.

\section{Recommendations}

Based on the results and conclusions the researchers suggest the following recommendations:

Since females' achievements were poor and also lower than their male counterparts, more effort is needed to fill this 
gender gap. It is, therefore, important and imperative for female students to develop their positive attitude towards the subject and make mathematics enjoyable, motivated for, and self - confident in learning it in order to enhance their performance.

Mathematics teachers are expected to teach mathematics in such a way that they should inculcate positive attitude in the students towards mathematics and to make them adaptable to mathematics learning consistently.

The school administrators should discuss the overall teaching learning process in learning mathematics with students, teachers, parents, and stakeholders and design ways through which students can get support.

School administrators, teachers, parents, and stakeholders should create conditions in which students learn mathematics with motivation, confidence, and enjoyment.

\section{References}

[1] E. Afari, "Examining the Factorial Validity of the Attitude towards Mathematics Inventory (ATMI) in the United Arab Emirates: Confirmatory Factor Analysis," International Review of Contemporary Learning Research-An International Journal, No. 1, 15-29, 2012.

[2] T., J. Ajai and I. B. Imoko, "Gender Differences in Mathematics Achievement and Retention Scores: A case of Problem-based Learning Method," International Journal of Research in Education and Science, Taraba State University, Nigeria, vol. 1, Issue 1, 2015.

[3] S. S. Ajay and M. B. Micah, "Sampling Techniques and Determination of Sample Size in Applied Statistics Research: An Overview," International Journal of Economics, Commerce and Management United Kingdom, vol. 11, Issue $11,2011$.

[4] Ajisuksmo, C. R. P., \& Saputri, G. R., "The Influence of Attitudes towards Mathematics, and Metacognitive Awareness on Mathematics Achievements," Creative Education, 8, 486497.

[5] Amelink, Catherine T., "Female Interest in Mathematics," Virginia Tech, SWE-AWE.

[6] Amin Burka, "Factors Affecting the Performance of Female students in Mathematics and Science: The case of selected High Schools in Shashemane, AAU, 2012.

[7] A. Bayaga and N. Wadesango, "Analysis of Students' Attitude on Mathematics Achievement-Factor Structure Approach," University of Fort Hare, South Africa, 2014.

[8] K. Asante, "Secondary students' attitudes towards mathematics," IFE Psychology IA, vol. 20, no. 1, pp. 121-133, 2012,2012

[9] Baharin, H. S., Othman, R. and Azizan, N. (2015). Statistical Analysis on the Determinants of Students' Academic Achievement: A study in UITM JOHOR. http://World conferences.net, 2015.

[10] R. Beaumont, "Multiple Linear Regressions”, 2010.

[11] R. Beaumont, "An Introduction to Statistics Correlation," 2012.
[12] Bereket Tesema and Kidus Meskele, "Binary Logistic Regression Analysis in Assessment and Identifying Factors that Influence Students' Academic Achievement: The Case of Natural and Computational Science," Wolaita Sodo University, Ethiopia, 2016.

[13] Boki Tola and Kebede Alemu, "Education System of Ethiopia (Mathematics and Science)," 2012.

[14] P. G. Brooks, “A Monte Carlo Program for Multiple Linear Regression," Ohio University, 2008.

[15] B. Celic and N. Koc., "Effect of Class Size on University Entrance Exam in Turkey," Procedia-Social and Behavioural Sciences, 919-924, 2015.

[16] C. Choi, "Junior Students' Attitude towards Mathematics in a Hong Kong secondary school," The University of Hong kong, 2015.

[17] N. Diem and H. T. Lapuente, "The steps to Follow in a Multiple Regression Analysis,” SAS Global Forum, pp-333, 2012.

[18] Eshetu Asfaw, Dilamo Otore, Tesfaye Ayele, and Zinabu Gebremariam, "Science and Mathematics Secondary Education in Ethiopia," Tunis, 2009.

[19] M. S. Farooq and S. Zia, "Students' Attitude towards Mathematics, "Pakistan Economic and Social Review, volume 46, No. 1, pp. 75-83, 2008.

[20] A. Field, "One-way Independent ANOVA," www.discoveringstatistics.com, 2016.

[21] Genene Bekele, "Attitude toward and Achievement of Natural Science and Mathematics Stream of Female Students in College of Teachers' Education in Oromia Region," Addis Ababa University, 2014.

[22] Getachew Bekele, "Anxiety, Attitude toward Mathematics and Mathematics Achievement of Tenth Grade Students at Government and Private Schools in Kolfe Keranio Sub City of Addis Ababa," Addis Ababa University, 2015.

[23] S. Georgiou, P. Stavrinides, and T. Kalavana," Is Victor better than Victoria at maths?" Educational Psychology in Practice, vol. 23, no. 4, pp. 329-342, 2007.

[24] J. Guthrie, and K. Knowles, "Promoting reading motivation, in Literacy and Motivation," Reading Engagement in Individuals and Groups," L. Verhoven and C. Snow, Eds., Lawrence Erlbaum Associates, Hillsdale, NJ, USA, 2001.

[25] M. K. Hannula," The Effect of Achievement, Gender and Classroom Context on Upper Secondary Students' Mathematical Beliefs, ” University of Turku, Finland, 2009.

[26] B. Hemmings, and R. Kay, "Prior achievement, effort, and mathematics attitude as predictors of current achievement," Australian Educational Researcher, vol. 37, no. 2, pp. 41-58, 2010.

[27] J. K. Amunga and A. M. Musasia, "Disparities in Mathematics Achievement among Secondary Schools: The Case of Kenya," Problems of Education in the $21^{\text {st }}$ Century, Volume 28, 2011.

[28] L. Kaster and A. Judy, "Gender Differences in Mathematics Attitudes in Coeducational and Single Sex Secondary Education," University of Sydney, 2016. 
[29] R. Kiamanesh, A. Mahdavi and M. Hezaveh, "Influential Factors Causing the Gender Differences in Mathematics Achievement Scores among Iranian Eight Graders on TIMSS 2003 data," Teacher Training University, Tehran, 2008.

[30] K. F. Nimon and F. L. Oswald, "Understanding the Results of Multiple Linear Regression Beyond Standardized Regression Coefficients," Organizational Research Methods, 16 (4), 650 674, 2013.

[31] S. M. Lindberg, T. S. Hyde, J. L. Petersen, and M. C. Linn, "New Trends in Gender and Mathematics Performance: a Meta Analysis," Psychological Bulletin, vol. 136, no. 6, pp. 1123-1135, 2010.

[32] M. Lovelace and P. Brickman, "Best Practice for Measuring Students' Attitude towards Learning Science," Department of Educational Psychology and Department of Biology, University of Georgia, Athens, vol. 12, 606-617, 2013.

[33] A. A. Majeed, N. G. Darmawan, and P. Lynch, "A confirmatory Factor Analysis of Attitude towards Mathematics Inventory (ATMI), " The university of Adelaide, Australia, vol. 15, No. 1, 121-135, 2013.

[34] M. L. Mata, V. Monteiro, F. Peixoto, "Attitudes towards Mathematics: Effects of Individual, Motivational, and Social Support Factors," Hindawi Publishing Corporation, 2012.

[35] J. K. Mensah, M. Okyyere, and M. Kuranchie, "Students Attitude towards Mathematics and Performance: Does the teacher attitude matter?" Journal of Education and Practice, vol. 4, No. 3, 2013.

[36] MOE, "Education System Ethiopia," /EP-Nuffic/ $2^{\text {nd }}$ Edition February, 2012

[37] MOE, "Education Statistics Annual Abstract," Ethiopia, 2013/14.

[38] L. Mohamed and H. Waheed," Secondary Students' Attitudes towards Mathematics in a Selected School Maldives," International Journal of Humanities and Science, Vol. 1, no. 15, pp. 277-281, 2011.

[39] S. Mubeen,, S. Saled, M. H. Arif, "Attitude towards Mathematics and Achievement in Mathematics among Secondary Level Boys and Girls, "Journal of Humanities and Social Science, vol. 6, Issue 4, pp 38-41, 2013.

[40] V. S. Mullis, O. M. Martin, G. E. Fierros, L. A. Goldberg, and E. S. Stemler, E., S., "Gender Differences in Achievement: IEA's Third International Mathematics and Science Study (TIMSS)," Educational Association for the Evaluation of Educational Achievement, Chestnut Hill, MA, 02467 USA, 2000.

[41] Mulugeta Atnafu, "Relation between Tenth Grade Students' Attitude and Components of Attitude in Algebra with Algebra Achievements of Addis Ababa Secondary Schools, Ethiopia. Mathematics Education, 9 (1), 57-72, Addis Ababa University, 2010.

[42] Mulugeta Atnafu, "Secondary School mathematics Teachers' Attitude in Teaching Mathematics," Addis Ababa University, Addis Ababa, Ethiopia, Mathematics Education, 9 (1), 57-72, 2014.

[43] F. Mushtaq, "Gender Difference in Mathematics Achievement," Karlstads Universitet, 2013.
[44] E. Mwenda, E. Gitaari, G. Nyaga, G. Muthaa, and G. Reche, "Factors Contributing to Students Poor Performance in Mathematics in Public Secondary Schools in Tharaka South District, Kenya," Journal of Education and Practice, vol. 4, No. 7, 2013.

[45] M. Nicolaidou, and G. Philippou, "Attitude toward Mathematics, Self-Efficacy and Achievement in ProblemSolving," European Research in Mathematics Education III, M. A. Mariotti, Ed., pp. 1-11, University of Pisa, Pisa, Italy, 2003.

[46] M. Paul and N. Hlanganipai, "The Influence of Students' Perceptions on Mathematics Performance: A Case of a Selected High School in South Africa," Mediterranean Journal of Social Sciences MCSER Publishing, Rome-Italy, Vol. 5 No3, 2014.

[47] K. Sanchez, L. Zimmerman, and R. Ye, "Secondary students' attitudes toward mathematics," Academic Exchange Quarterly, vol. 8, no. 2, pp. 56-60, 2004.

[48] T. Scafidi and K. Bui, "Gender similarities in math performance from middle school through high school," Journal of Instructional Psychology, vol. 37, no. 3, pp. 252$255,2010$.

[49] Sileshi Zeleke, "Gender Differences in Mathematics Performance in the Elementary Grades: Implications for Women's Participation in Scientific and Technical Occupations," Addis Ababa University, 2001.

[50] Singh, K., Granville, M. and Dika, S. (2002). Mathematics and science achievement: effects of motivation, interest, and academic engagement, Journal of Educational Research, vol. 95, no. 6, pp. 323-332, 2002.

[51] Skaalvik, S. and Skaalvik, E. M., "Gender differences in math and verbal self-concept, performance expectations, and motivation," Sex Roles, vol. 50, no. 3-4, pp. 241-251, 2004.

[52] M. Tapia, "An Instrument to Measure Mathematics Attitudes,” Berry College, GA mtapia@berry.edu, volume 8, Issue 2, 2004.

[53] R. Taylor, "Interpretation of the Correlation Coefficient: Basic Review," Journal of Diagnostic Medical Sonography, Vol. 6, JDMS 1: 35-39, 1990.

[54] A. Tella, "The impact of Motivation on Student's Academic Achievement and Learning Outcomes in Mathematics among Secondary School Students in Nigeria," Eurasia Journal of Mathematics, Science and Technology Education, 3 (2), 149156, 2007.

[55] A. Palacios, V. Arias, and B. Arias, "Attitudes towards Mathematics: Construction and Validation of a Measurement Instrument," Revista de Psicodidactica, 19 (1), 67-91, 2014.

[56] www.foxitsoftware.com, "Understanding the Independent ttest," Retrieved on 04-12-2013, version 1.0.0.412, 2013.

[57] L. Kyei, B. Apam, and K. S. Nokoe, "Some Gender differences in Performance in Senior High Mathematics Examinations in Mixed High Schools," American Journal of Social and Management Sciences, ISSN print, 2156-1540, 2011.

[58] I. Michael, "Factors Leading to the Poor Performance in Mathematics Subject in Kibaha Secondary Schools," The open University of Tanzania, 2015. 
[59] B. Gegbe, A. Sundai, and V. K. Sheriff, "Factors Contributing to Students' Poor Performance in Mathematics at West African Senior School Certification Examination (A Case Study: Kenema City, Eastern Province Sierra Leone," International Journal of Engineering Research and General Science, Volume 3, Issue 2, 2015.
[60] Zeneba Lemesa, "Factors that Affect Students' Academic Achievement in Government Secondary Schools of Asella Town," Oromia National Regional State, Ethiopia, 2015.

[61] PISA, "Student Learning: Attitudes, Engagement and Strategies," OECD Learning for Tomorrow's World-2004. 